\title{
Obstructive Sleep Apnea and Testosterone Replacement Therapy
}

\author{
Sandro La Vignera, ${ }^{1, *}$ Aldo E. Calogero, ${ }^{1}$ Rossella Cannarella, ${ }^{1}$ Rosita A. Condorelli, \\ Cristina Magagnini, ${ }^{1}$ and Antonio Aversa ${ }^{2}$
}

\begin{abstract}
The evidence on the role of obstructive sleep apnea (OSA) in the pathogenesis of hypogonadism and the impact of testosterone replacement therapy (TRT) in OSA patients are still contradictory. OSA is generally considered to be a relative contraindication as TRT is feared to worsen sleep apnea so that ventilatory capacity should be strictly investigated in advance and monitored thereafter. Few controlled studies have been released on the long-term effects of TRT in patients with OSA due to methodological limitations at study entry. Data from recent randomized placebo-controlled studies show a time-dependent influence on nocturnal hypoxia, and a positive impact after a longer time of exposure in selected patients. Since these results await further confirmation from larger studies, we suggest to use TRT cautiously in obese hypogonadal patients with hypoventilatory syndrome, especially if they are not on continuous positive airway pressure treatment.
\end{abstract}

Keywords: obstructive sleep apnea; hypogonadism; testosterone; testosterone replacement therapy

\section{Introduction}

Sleep disorders are clinical conditions that worsen the quality of life and prognosis. ${ }^{1}$ They include a number of diseases characterized by abnormal breathing during sleep, due to a shrinkage or obstruction of the upper airways. One of the most common forms of sleep disorders is obstructive sleep apnea (OSA). ${ }^{2}$ OSA is characterized by repetitive and intermittent, partial or complete collapse of the upper airway during sleeping, regardless of the presence of daytime symptoms. This happens because upper airway dilator muscles fail to counter the negative pressure in the airways during inspiration. This effect is present during sleep because an enhanced muscular activity is lost. If symptoms are present, this condition is called "OSA syndrome" (OSAS) and the main presentations are sleep fragmentation with breathing interruptions, decreased sleep time, shorter REM time, loud snoring, and daytime sleepiness. This leads to oxygen $\left(\mathrm{O}_{2}\right)$ desaturation with hypoxemia and hypercapnia. ${ }^{3}$ Over 50 years, $\sim 1-2 \%$ of women and $2-4 \%$ of men have OSA. ${ }^{4}$ OSA severity is classified by measuring the "apneahypopnea index" (AHI). An "apneic event" is defined by a decrease of airflow by $>90 \%$ lasting for at least $10 \mathrm{sec}$; meanwhile hypopnea is defined as a decrease of airflow from $30 \%$ to $90 \%$ with $3 \%$ or more $\mathrm{O}_{2}$ desaturation. On the basis of the number of "apneahypopnea" events during sleeping, three OSA levels can be identified: normal (AHI $\leq 5)$, mild $(5<$ AHI $<15$ ), moderate $(15 \leq \mathrm{AHI}<30$ ), and severe (AHI $\geq 30) .^{2}$ Etiopathogenesis of OSAS implies several factors, the main being obesity due to fatty deposits in the upper airways.

OSA is an independent risk factor for several diseases. These include cerebrocardiovascular diseases (hypertension, myocardial infarction, congestive heart failure, arrhythmias such as atrial fibrillation, and stroke), metabolic diseases (type 2 diabetes mellitus),

\footnotetext{
${ }^{1}$ Department of Clinical and Experimental Medicine, University of Catania, Catania, Italy

2Department of Experimental and Clinical Medicine, "Magna Graecia" University, Catanzaro, Italy.

*Address correspondence to: Sandro La Vignera, MD, PhD, Department of Clinical and Experimental Medicine, University of Catania, Policlinico “G. Rodolico," via S. Sofia 78, Catania 95123, Italy, Email: sandrolavignera@unict.it
}

(C) Sandro La Vignera et al. 2020; Published by Mary Ann Liebert, Inc. This Open Access article is distributed under the terms of the Creative Commons License (http://creativecommons.org/licenses/by/4.0), which permits unrestricted use, distribution, and reproduction in any medium, provided the original work is properly cited. 
and depression. Recently, hypoxia has been suggested as possibly involved in the pathogenesis of pituitarygonadal axis alterations, leading to obesity-related hypogonadotropic hypogonadism. ${ }^{3}$ The evidence on the impact of testosterone replacement therapy (TRT) on OSAS is still contradictory. The aim of this review was to analyze the impact of OSA in the pathogenesis of hypogonadism and to critically examine the pros and cons of TRT in hypogonadal patients with OSA. To accomplish this, we performed a search on Pubmed, Science-direct, Ovid, and Scopus, using the following keywords: obstructive sleep apnea, OSA, hypogonadism, testosterone replacement therapy, and TRT. Particularly, special attention was given to evidence coming from randomized controlled study design unbiased by improperly high TRT dosages.

\section{The Role of OSA in the Pathogenesis of Hypogonadism}

A strong relationship between serum testosterone levels and sleep disorders such as OSA has been shown. Testosterone synthesis is regulated by the hypothalamic-pituitary-testicular axis and it relies on luteotropic hormone and follicle-stimulating hormone pulsatile secretion. Serum testosterone levels change during the day: they peak on the early morning upon awakening and reach the nadir at the end of the day. ${ }^{5-7}$ Accordingly, plasma testosterone levels raise with the sleep onset, they then have a spike at the first REM sleep episode, and continue to rise until awakening. Testosterone levels decrease when REM latency is longer, such as in old age and in sleep disorders. The sleep fragmentation disrupts testosterone rhythm.,

OSAS is associated with a decreased gonadal function, because of less REM sleep episodes and major sleep fragmentation, which contribute to an alteration of gonadotrophin-releasing hormone pulsatility, ${ }^{8}$ and this is confirmed by negative correlation between AHI score serum testosterone levels. ${ }^{3}$ Further proof is given by the $\mathrm{O}_{2}$ desaturation index and by $\mathrm{O}_{2}$ nadir, both related with testosterone decrease.

Obesity may play a role in the correlation between OSAS and hypogonadism. Accordingly, the aromatase enzyme, expressed in adipocytes, converts testosterone into $17 \beta$-estradiol. The latter exerts its negative feedback on gonadotropin secretion and leads to testosterone decrease. The alteration of testosterone/ $17 \beta$-estradiol ratio brings to a compensatory synthesis of sex-hormone binding glubulin, which binds testosterone in the bloodstream. Intermittent hypoxia and repetitive short cycles of desaturation and reoxygenation are all involved in white adipose tissue proliferation and pathophysiological processes, leading to OSAS, that is, overproduction of cytokines, chemokines, and adipokines. An increase of T cells, B cells, macrophages, leukocytes, mast cell, and neoangiogenesis mechanisms has been observed in fat tissue of obese patients. Macrophages infiltrate adipocytes and worsen tissue inflammation and insulin resistance. T cells and $B$ cells change their phenotype from M2 to M1proinflammatory, which produces proinflammatory adipokines, such as tumor necrosis factor- $\alpha$, interleukin (IL)-6, IL-1 $\beta$, and leptin. Local tissue hypoxia leads to the activation of hypoxia-inducible factor, which triggers the expression of vascular endothelial growth factor and plasminogen activator inhibitor$1 .^{8}$ Moreover, obesity worsens hypotestosteronemia. The body mass index is considered the primary determinant of testosterone levels in men with OSA $^{5,6,9}$ and it is associated with increased severity of the disorder. These data are further confirmed by the linear correlation shown between weight loss and increased plasma testosterone in obese men (Fig. 1). ${ }^{10}$

Low testosterone levels are strictly correlated with the presence both physical and mental fatigue and decreased physical capacity in OSA patients. ${ }^{3}$ Bercea et al. found that reduced physical activity and physical fatigue are related with serum testosterone and not with $\mathrm{O}_{2}$ saturation. Therefore, low serum testosterone is an independent predictor of this condition in OSA patients. ${ }^{7}$ According to erectile dysfunction (ED) definition, ${ }^{11}$ this is recognized as the main symptom that forces OSAS patients to seek help. ${ }^{2,12}$ It is especially evident in patients with severe OSA and less evident in patients with mild or moderated OSA.,13,14 A recent meta-analysis showed that patients with OSA have a relative risk of 1.82 to develop ED. A study conducted by Gonçalves et al. reported a prevalence of $48 \%$ of ED in patient with severe OSA ${ }^{15}$; the severity of OSA is considered to be an important factor for the development of ED. ${ }^{2,16}$ Phosphodiesterase type 5 inhibitors (PDE5is) on demand are useful as a treatment for ED in this subgroup of patients, with high satisfaction rates and no contraindication reported. ${ }^{17}$

\section{TRT and OSA}

Historically, TRT has been considered dangerous for patients with severe OSAS because it could exacerbate symptoms, increasing $\mathrm{AHI}$, decreasing $\mathrm{O}_{2}$ saturation, and interfering with central response to hypo- and 


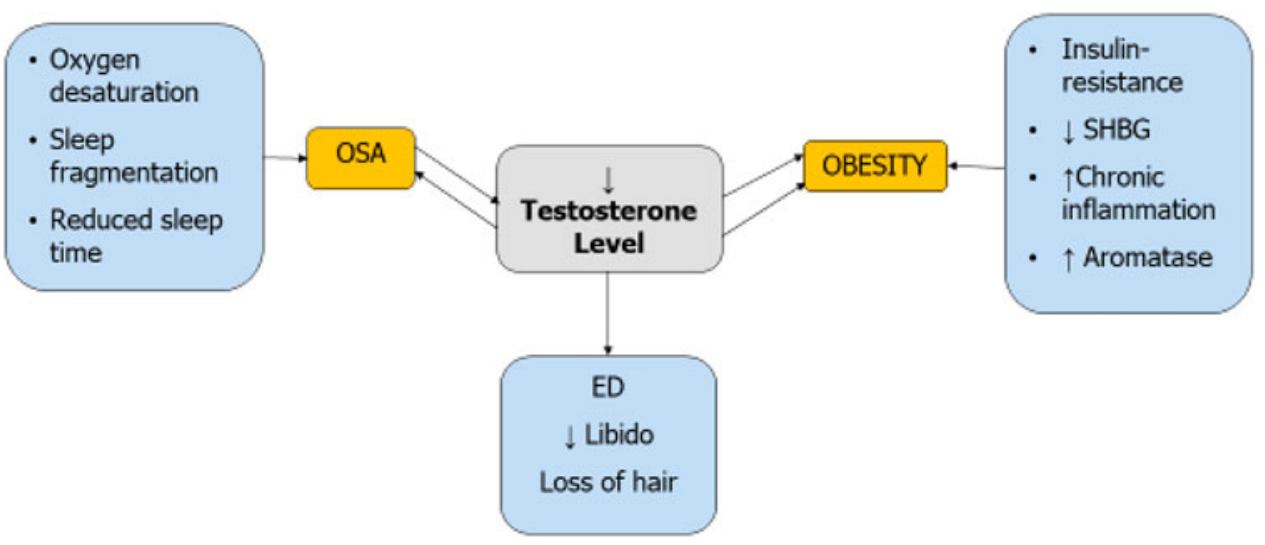

FIG. 1. Two-sided relationship between OSA, obesity, and testosterone level. OSAS and obesity contribute to reduce testosterone level in bloodstream; at the same time, lower testosterone level worsens obesity and sleep disorders. OSA, obstructive sleep apnea; OSAS, OSA syndrome.

hypercapnia., ${ }^{5,6}$ The physiological mechanisms that are supposed to interfere with OSA are (1) reduced contraction of airway dilator muscles and subsequent collapse of the upper airways, (2) increased metabolic consumption with greater $\mathrm{O}_{2}$ demand and subsequent hypoxia and polycythemia, and (3) reduced neural central response to hypoxia and hypercapnia, with increase in the number of apneas and hypopneas and the rela- tive AHI. However, TRT does not influence upper airways size or the Epworth Sleepness Scale. Nevertheless, it is a cause of reduced total time slept and of increased disrupted sleeping and duration of hypoxia (Fig. 2). ${ }^{18}$

Current guidelines recommend TRT for adult men with severely reduced testosterone levels $(<9.2 \mathrm{nmol} / \mathrm{L}),{ }^{19}$ but they suggest against its use in patients with untreated severe OSA since treating hypogonadal patients may

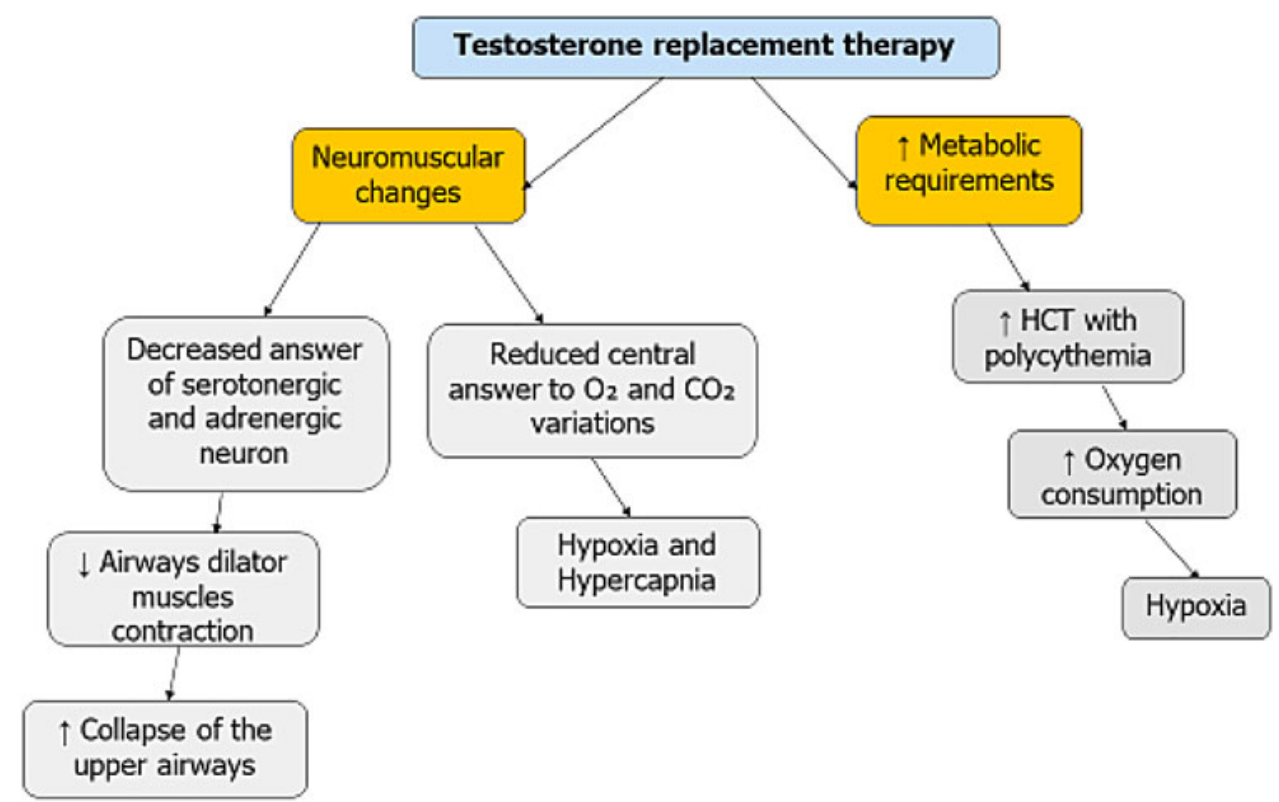

FIG. 2. Testosterone replacement therapy worsens OSA symptoms through neuromuscular changes and raised metabolic requirements. This condition causes collapse of the upper airway and onset of hypoxia. 
cause an increase of apneas and hypopneas events. ${ }^{2}$ Actually, few studies have considered the effect of TRT in patients with OSAS. In one of them, testosterone given IM was compared with placebo and it has been shown that hypoxic ventilatory drive significantly decreased in TRT patients, whereas hypercapnoic ventilatory drive did not significantly change. ${ }^{20}$ However, this 6-week-long prospective study examined only five hypogonadal patients, reporting the development of OSA in one case and no change in respiratory parameters in the latter patients. ${ }^{20}$ Overall, the evidence provided by this study is very low due to the small duration and sample size. Similarly, a randomized placebo-controlled double-blind study on 17 patients without OSA analyzed the effects of high testosterone dosage on the quality of sleep. Particularly, the TRT scheme consisted of $500 \mathrm{mg}$ IM administered in the first week, $250 \mathrm{mg}$ in the second, and $250 \mathrm{mg}$ in the third week. Unsurprisingly, TRT at high doses showed to worsen total time slept and to increase the duration of hypoxia and to disrupt breathing during sleep. ${ }^{21}$ This proved the negative effects of TRT on ventilatory function when a high dosage is chosen. However, the adverse effects of high-dose TRT have already been ascertained. ${ }^{19}$

A prospective cohort study carried out in 3422 male U.S. military service members, retired and dependents aged 40-64 years, analyzed the risk of developing TRTdependent adverse events in a 17-month-long observation. Patients on TRT had a reduced risk of coronary artery disease with an improved cardiovascular eventfree survival. By contrast, the risk of OSA development was higher in TRT users than in controls $(16.5 \%$ vs. $12.7 \%) .{ }^{22}$ Thus, starting TRT in patients with no previous sign of OSA may prompt to its development. This could be due to the exacerbation of polycythemia. ${ }^{23}$ For this reason, TRT in OSA patients must be monitored by polysomnography and, potentially, treated with continuous positive airway pressure (CPAP); in patients who are not responsive to CPAP, TRT dosage must be lowered or withdrawn. ${ }^{24,25}$

But what about the effects of TRT in hypogonadal patients with OSAS? Four randomized placebocontrolled clinical trials have been performed on this matter so far. The study by Shigehara et al. has been performed in 48 OSA patients with hypogonadism who underwent TRT. Interestingly, the authors reported an improvement of sleep disturbances compared with placebo at the 12th month of TRT. ${ }^{26}$ This represents the longest trial carried out so far, since other studies showed a duration ranging from 12 to
18 weeks. After 12 weeks, TRT (testosterone undecanoate was administered in a randomized placebocontrolled study design at the dose of $1000 \mathrm{mg}$ at weeks 0,6 , and 12) and did not show to influence sleep quality in 67 obese patients with OSA. ${ }^{27}$ Another study carried out in 21 obese patients with OSA and hypogonadism treated with testosterone undecanoate (1000 mg at weeks 0,6 , and 12) reported a positive correlation between serum testosterone levels and hyperoxic ventilator recruitment threshold, correlating with time of oxygen desaturation during sleep at 7 weeks but not at 18 weeks. ${ }^{28}$ Similarly, another randomized placebo-controlled trial in 67 obese patients with OSA and hypogonadism found that TRT worsens oxygen saturation at week 7 . No effect of TRT on sleep time with oxygen saturation $<90 \%$ was observed after 18 weeks. ${ }^{29}$ Taken together, these findings highlight that the influence of TRT on nocturnal oxygen saturation in OSA patients is time dependent. ${ }^{30}$ Particularly, although after 7 weeks, a negative impact seems to occur, no effect has been registered after 12 and 18 weeks and even a positive effect has been described after 12 months. ${ }^{31}$ However, further prospective studies on larger sample size and with longer duration are needed to confirm this initial evidence.

\section{Conclusion}

In summary, this review summarizes the evidence on the mechanisms involved in the pathogenesis of hypogonadism in patients with OSAS, such as abnormal circadian rhythm of gonadotrophin secretory patterns associated with obesity. TRT may represent a risk factor for OSA development and therefore, respiratory function monitoring is recommended especially in obese patients during TRT. Scanty evidence has been released on the effect of TRT in patients with OSA. Data from recent randomized placebo-controlled studies address to TRT a time-dependent influence on nocturnal hypoxia, showing a positive impact after a longer time of exposure. Also, CPAP and PDE5i can be considered safe procedures to ameliorate sexuality in hypogonadal patients with OSA. We suggest to use TRT cautiously in obese hypogonadal patients with hypoventilatory syndrome especially if they are not on CPAP. The latter aspect needs to be further confirmed by larger controlled studies.

\section{Author Disclosure Statement}

No competing financial interests exist. 


\section{Funding Information}

No funding was received for this article.

\section{References}

1. Kazuyoshi S, Hiroyuki K, Kazuhiro S, et al. Sleep disturbance as a clinical sign for severe hypogonadism: Efficacy of testosterone replacement therapy on sleep disturbance among hypogonadal men without obstructive sleep apnea. Aging Male. 2018;21(2):99-105.

2. Cho YW, Kim KT, Moon HJ, et al. Comorbid insomnia with obstructive sleep apnea: Clinical characteristics and risk factors. J Clin Sleep Med. 2018;14(3):409-417.

3. Foti DP, Brunetti A. Editorial: "linking hypoxia to obesity." Front Endocrinol (Lausanne) 2017;8:34.

4. Maspero C, Giannini L, Galbiati G, Rosso G, Farronato G. Obstructive sleep apnea syndrome: A literature review. Minerva Stomatol. 2015;64(2):97109.

5. Wittert $\mathrm{G}$. The relatioship between sleep disorders and testosterone. Curr Opin Endocrinolo Diabetes Obes. 2014;21(3):239-243.

6. Wittert $G$. The relationship between sleep disorders and testosterone in men. Asian J Androl. 2014;16(2):262-265.

7. Bercea RM, Mihaescu T, Cojocaru C, Bjorvatn B. Fatigue and serum testosterone in obstructive sleep apnea patients. Clin Respir J. 2015;9(3): 342-349.

8. Messineo S, Laria AE, Arcidiacono B, et al. Cooperation between HMGA1 and HIF-1 contributes to hypoxia-induced VEGF and Visfatin gene expression in 3T3-L1 adipocytes. Front Endocrinol (Lausanne) 2016;7:73.

9. Macrea MM, Martin Tj, Zagrean L. Infertility and obstructive sleep apnea: The effect of continuous positive airway pressure therapy on serum prolactin levels. Sleep Breath. 2010;14(3):253-257.

10. Donini LM, Cuzzolaro M, Gnessi L, et al. Obesity treatment: Results after 4 years of a nutritional and psycho-physical rehabilitation program in an outpatient setting. Eat Weight Disord. 2014;19(2):249-260.

11. Feldman HA, Goldstein I, Hatzichrisou DG, Krane RJ, McKinlay JB. Impotence and its medical and psychosocial correlates: Result of Massachusetts Male Aging Study. J Urol. 1994;151(1):54-61.

12. Lue FT. Erectile dysfunction. N Engl J Med. 2000;342(24):1802-18013.

13. Margel D, Cohen M, Livne PM, Pillar G. Severe, but not mild, obstructive sleep apnea syndrome is associated with erectile dysfunction. Urology. 2004;63(3):545-549.

14. Liu L, Kang R, Zhao $S$, et al. Sexual dysfunction in patient with obstructive sleep apnea: A systemic review and meta-analysis. J Sex Med. 2015; 12(10):1992-2003.

15. Gonçalves MA, Guilleminault C, Ramos E, et al. Erectile dysfunction, obstructive sleep apnea syndrome and nasal CPAP treatment. Sleep Med. 2005;6(4):333-339.

16. Omar B, Jing W. Testosterone deficiency and sleep apnea. Sleep Med Clin 2016;11(4):525-529.

17. Campos-Juanatey F, Fernandez-Barriales $M$, Gonzalez M, Portillo-Martin JA. Effects of obstructive sleep apnea and its treatment over the erectile function: A systematic review. Asian J Androl. 2017;19(3):303-310.

18. Hanafy HM. Testosterone therapy and obstructive sleep apnea: Is there a real connection? J Sex Med. 2007:4(5):1241-1246.

19. Bhasin S, Brito JP, Cunningham GR, et al. Testosterone therapy in men with hypogonadism: An Endocrine Society Clinical Practice Guideline. J Clin Endocrinol Metab. 2018;103(5):1715-1744.

20. Matsumoto AM, Sandblom RE, Schoene RB, et al. Testosterone replacement in hypogonadal men: Effects on obstructive sleep apnea, respiratory drives and sleep. Clin Endocrinol. 1985;22(6):713-721.

21. Liu PY, Yee B, Wishart SM, et al. The short-term effects of high-dose testosterone on sleep, breathing, and function in older men. J Clin Endocrinol Metab. 2003;88(8):3605-3613.

22. Cole AP, Hanske J, Jiang W, et al. Impact of testosterone replacement therapy on thromboembolism, heart disease and obstructive sleep apnoea in men. BJU Int. 2018;121(5):811-818.

23. Zelveian PA, Dgerian LG. The main pathophysiological mechanisms of kidney injury in obstructive sleep apnea syndrome. Ter Arkh. 2014;86(6): 100-105.

24. Choi JB, Loredo JS, Norman D, et al. Does obstructive sleep apnea increase hematocrit? Sleep Breath. 2006;10(3):155-160.
25. Bachman E, Travison TG, Basaria S, et al. Testosterone induces erythrocitosis via increased erythropoietin/hemoglobin set point. J Gerontol A Biol Sci Med Sci. 2014;69(6):725-735.

26. Shigehara K, Konaka H, Sugimoto K, et al. Sleep disturbance as a clinical sign for severe hypogonadism: Efficacy of testosterone replacement therapy on sleep disturbance among hypogonadal men without obstructive sleep apnea. Aging Male. 2018;21(2):99-105.

27. Melehan KL, Hoyos CM, Yee BJ, et al. Increased sexual desire with exogenous testosterone administration inmen with obstructive sleep apnea: A randomized palcebo-controlled study. Andrology. 2016;4(1): 55-61.

28. Killick R, Wang D, Hoyos CM, Yee BJ, Grunstein RR, Liu PY. The effects of testosterone on ventilatory responses in men with obstructive sleep apnea: A randomised, placebo-controlled trial. J Sleep Res. 2013;22(3): 331-336.

29. Hoyos CM, Killick R, Yee BJ, Grunstein RR, Liu PY. Effects of testosterone therapy on sleep and breathing in obese men with severe obstructive sleep apnoea: A randomized placebo-controlled trial. Clin Endocrinol (Oxf). 2012;77(4):599-607.

30. Kim SD, Cho KS. Obstructive sleep apnea and testosterone deficiency. World J Mens Health. 2019;37(1):12-18.

31. Pastore AL, Palleschi G, Ripoli A, et al. Severe obstructive sleep apnoea syndrome and erectile dysfunction: A prospective randomised study compare sildenafil vs nasal continuous positive airway pressure. Int J Clin Pract. 2014:68(8):995-1000.

Cite this article as: La Vignera $S$, Calogero $A E$, Cannarella $R$, Condorelli RA, Magagnini C, Aversa A (2020) Obstructive sleep apnea and testosterone replacement therapy. Androgens: Clinical Research and Therapeutics 1.1, 10-14, DOI: 10.1089/andro.2020.0001.

Publish in Androgens: Clinical Research and Therapeutics

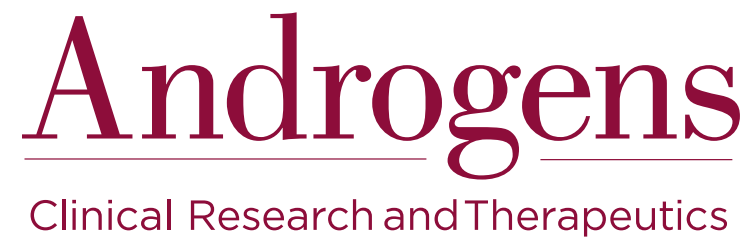

liebertpub.com/andro 